\title{
Metastatic colorectal cancer in the elderly: An overview of the systemic treatment modalities (Review)
}

\author{
RAFAŁ STEC ${ }^{1}$, LUBOMIR BODNAR ${ }^{1}$, MARTA SMOTER ${ }^{1}$, \\ MICHAŁ MĄCZEWSKI ${ }^{2}$ and CEZARY SZCZYLIK ${ }^{1}$ \\ ${ }^{1}$ Department of Oncology, Military Institute of Medicine; ${ }^{2}$ Department of Clinical Physiology, \\ Medical Center of Postgraduate Education, Warsaw, Poland
}

Received July 22, 2010; Accepted October 26, 2010

DOI: $10.3892 / \mathrm{ol} .2010 .212$

\begin{abstract}
Colorectal cancer (CRC) is one of the most frequently occurring types of cancer. Worldwide, more than 800,000 new cases of CRC are diagnosed each year. The median ages at CRC diagnosis and death are 71 and 75 years, respectively. The majority ot patients (50-60\%) with colorectal cancer are diagnosed at stage IV disease. Patients aged 65 or older are characterized by a higher incidence of significant co-morbidities, decreased regenerative capacity of bone marrow and worse general performance. Anti-neoplastic therapies used for the treatment of colorectal cancer include irinotecan, oxaliplatin, 5-fluorouracil, leucovorin, capecitabine and monoclonal antibodies. Analysis of the efficacy of the presented chemotherapeutic and chemoimmunotherapeutic regimens in the treatment of metastatic CRC in patients older than 65 and 70 years compared to 'younger' patients, generally demonstrated comparable efficacy, time to disease progression and overall survival. Age criterion should not be considered when assessing the eligibility of patients with metastatic CRC for treatment of the above-mentioned chemotherapeutic and chemoimmunotherapeutic regimens. Treatment should be individualized based on the potential risks and benefits anticipated for each patient.
\end{abstract}

\section{Contents}

1. Introduction

2. Toxicity and efficacy of chemotherapy in patients older than 65 years

3. Chemotherapy

4. Targeted therapies

5. Conclusions

Correspondence to: Dr Rafał Stec, Department of Oncology, Military Institute of Medicine in Warsaw, 128 Szaserów Street, 04-141 Warsaw, Poland

E-mail:drrafals@wp.pl

Key words: colorectal cancer, chemotherapy, targeted therapies, efficacy, toxicity, elderly patients

\section{Introduction}

Colorectal cancer (CRC) is one of the most common types of cancer. Worldwide, over 800,000 new cases of CRC are diagnosed each year (1). The incidence of CRC increases with age in subjects more than 40 years of age, while a rapid increase in incidence is found in individuals older than 50 years of age. The median ages at CRC diagnosis and death are 71 and 75 years, respectively (2).

The majority of patients (50-60\%) with colorectal cancer are diagnosed at stage IV of the disease (3). Palliative chemotherapy is the only therapeutic option currently available. It provides patients with the opportunity of prolonged survival and an improvement in the quality of life. Anti-neoplastic therapies used for the treatment of CRC include irinotecan, oxaliplatin, 5-fluorouracil, leucovorin and capecitabine as well as monoclonal antibodies, bevacizumab, panitumumab and cetuximab.

Therapeutic decisions involving patients above 65 years of age (currently the largest group among colorectal cancer patients) are a serious issue in oncology. This group is characterized by a higher incidence of significant co-morbidities (cardiovascular disorders, metabolic disorders, decreased glomerular filtration rate and liver disorders), decreased regenerative capacity of bone marrow (higher incidence and intensity of hematological complications of chemotherapy) as well as worse general performance.

\section{Toxicity and efficacy of chemotherapy in patients older than 65 years}

In their study regarding the pharmacology of cytotoxic agents used in oncology, Lichtman and Villani (4) presented differences in the toxicity profile and grade between younger and older patients. Antimetabolite 5-fluorouracil was found to cause stomatitis in patients $\geq 70$ years of age compared to younger subjects (19 and 11\%, respectively). The fluoropyrimidine derivative capecitabine, administered orally, is characterized by a specific toxicity profile, manifesting as hand-foot syndrome for which the intensity and incidence is age-associated. The topoisomerase I inhibitor, irinotecan, used in elderly patients is associated with a higher incidence and toxicity grade of diarrhea. 
Table I. Toxicity of ILF and LF regimens in patients younger and older than 70 years of age ${ }^{\text {a }}$

\begin{tabular}{lrrrrrr}
\hline & \multicolumn{2}{c}{ Patients $<70$ years of age } & & \multicolumn{2}{c}{ Patients $\geq 70$ years of age } & \\
\cline { 2 - 3 } Toxicities of grade $\geq 3(\%)$ & ILF & LF & & ILF & LF & P-value \\
\hline Leucopenia & 16.9 & 7.0 & & 18.5 & 6.4 & 0.850 \\
Neutropenia & 28.9 & 16.1 & & 29.7 & 19.9 & 0.180 \\
Thrombocytopenia & 0.5 & 0.7 & & 1.2 & 0.7 & 0.830 \\
Nausea & 11.3 & 5.8 & & 10.8 & 3.7 & 0.280 \\
Diarrhea & 20.5 & 11.4 & & 23.4 & 12.6 & 0.330 \\
Vomiting & 9.6 & 5.3 & & 9.7 & 2.5 & 0.190 \\
Stomatitis & 2.5 & 2.6 & & 4.0 & 3.6 & 0.240 \\
Hand-foot syndrome & 1.0 & 1.5 & & 1.7 & 2.3 & 0.290 \\
Hepatotoxicity & 4.6 & 1.7 & & 9.8 & 7.7 & 0.024 \\
Infection without neutropenia & 1.2 & 1.1 & 2.4 & 2.6 & 0.350 \\
Thrombosis & 4.9 & 4.2 & 4.3 & 4.5 & 0.970 \\
\hline
\end{tabular}

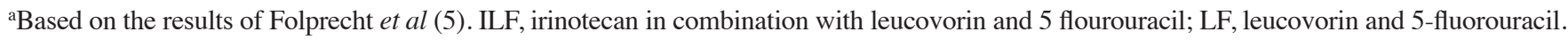

Table II. Efficacy of ILF and LF regimens in patients younger and older than 70 years of age .

\begin{tabular}{|c|c|c|c|c|}
\hline & \multicolumn{2}{|c|}{ Patients $<70$ years of age } & \multicolumn{2}{|c|}{ Patients $\geq 70$ years of age } \\
\hline & ILF & $\mathrm{LF}$ & ILF & $\mathrm{LF}$ \\
\hline Median time to disease progression (months) & $\begin{array}{c}8.2 \\
(n=776)\end{array}$ & $\begin{array}{c}6.3 \\
(n=1308)\end{array}$ & $\begin{array}{c}9.2 \\
(\mathrm{n}=220)\end{array}$ & $\begin{array}{c}7.0 \\
(n=376)\end{array}$ \\
\hline P-value & \multicolumn{2}{|c|}{$<0.0001$} & \multicolumn{2}{|c|}{0.0026} \\
\hline Median overall survival (months) & $\begin{array}{c}17.1 \\
(\mathrm{n}=765)\end{array}$ & $\begin{array}{c}14.7 \\
(\mathrm{n}=1308)\end{array}$ & $\begin{array}{c}17.6 \\
(\mathrm{n}=219)\end{array}$ & $\begin{array}{c}14.2 \\
(\mathrm{n}=375)\end{array}$ \\
\hline P-value & \multicolumn{2}{|c|}{$<0.0003$} & \multicolumn{2}{|c|}{0.1500} \\
\hline Complete response rate (\%) & $\begin{array}{c}46.6 \\
(n=745)\end{array}$ & $\begin{array}{c}29 \\
(\mathrm{n}=1218)\end{array}$ & $\begin{array}{c}50.5 \\
(\mathrm{n}=208)\end{array}$ & $\begin{array}{c}30.3 \\
(\mathrm{n}=346)\end{array}$ \\
\hline P-value & \multicolumn{2}{|c|}{$<0.0001$} & \multicolumn{2}{|c|}{0.0001} \\
\hline
\end{tabular}

aBased on the results of Folprecht et al (5). ILF, irinotecan in combination with leucovorin and 5 flourouracil; LF, leucovorin and 5-fluorouracil.

\section{Chemotherapy}

Irinotecan. Folprecht et al (5) evalutated 2691 patients treated with irinotecan in combination with leucovorin and 5-fluorouracil (ILF regimen) compared to leucovorin and 5-fluorouracil (LF regimen) as first-line therapy for metastatic colorectal cancer. Toxicity and effectiveness were compared in the two groups of patients: below and above 70 years of age. A total of 599 (22.3\%) patients were $\geq 70$ years of age, including 185 (6.9\%) patients $\geq 75$ years of age and $1 \%>80$ years.

No differences in effectiveness were found for the ILF regimen between the age groups $<70$ and $\geq 70$. The objective response rate was 46.6 and $50.5 \%$, respectively; median time to disease progression was 8.2 and 9.2 months, respectively, and median overall survival was 17.1 and 17.6 months, respectively. Effectiveness of the LF regimen was reduced, but no differences were found between the age groups $<70$ and $\geq 70$ years. The objective response rate was 29 and $30.3 \%$, respectively; median time to disease progression was 6.3 and 7.0 months, respectively, and median overall survival was 14.7 and 14.2 months, respectively. The results are shown in Table II.

Addition of irinotecan to the chemotherapy resulted in a significant increase in the incidence of grade 4 toxicity (leucopenia, neutropenia, diarrhea, nausea and vomiting) in the two patient groups. However, no differences in toxicity were found between the age groups $<70$ and $\geq 70$ years apart from hepatotoxicity which was more common in the elderly patients. Analysis of the $\geq 70$ years of age subgroup revealed a higher incidence of grade 4 neutropenia vs. the $<70$ years of age subgroup (24.3 and $16.1 \%$, respectively) among the patients treated with the LF regimen. These results are shown in Table I. 
Table III. Assessment of response to capecitabine chemotherapy and a FOLFIRI regimen in patients above 65 years of age $(\mathrm{n}=123)^{\mathrm{a}}$.

\begin{tabular}{|c|c|c|c|c|c|c|}
\hline & \multicolumn{2}{|c|}{$\begin{array}{l}\text { Capecitabine } \\
(\mathrm{n}=56)\end{array}$} & \multicolumn{2}{|c|}{$\begin{array}{c}\text { FOLFIRI } \\
(\mathrm{n}=67)\end{array}$} & \multirow[b]{2}{*}{$\chi^{2}$} & \multirow[b]{2}{*}{ P-value } \\
\hline & $\mathrm{n}$ & $\%$ & $\mathrm{n}$ & $\%$ & & \\
\hline Overall response $\mathrm{b}^{\mathrm{b}}$ & 8 & 16.4 & 18 & 28.1 & 2.18 & 0.1398 \\
\hline Complete response & 2 & 4.1 & 6 & 9.4 & $0.51^{\mathrm{c}}$ & $0.4733^{c}$ \\
\hline Partial response & 6 & 12.3 & 12 & 18.7 & 0.88 & 0.3491 \\
\hline Stable disease & 28 & 57.1 & 35 & 54.7 & 0.07 & 0.7945 \\
\hline Progressive disease & 13 & 26.5 & 12 & 17.2 & 1.45 & 0.2288 \\
\hline Not evaluable & 7 & 12.5 & 2 & 3.0 & & \\
\hline
\end{tabular}

${ }^{\mathrm{a} B a s e d}$ on the results by Stec et al (10). ${ }^{\mathrm{b}}$ Complete plus partial response. ${ }^{\mathrm{c}}$ Values calculated by the Chi-square $\left(\chi^{2}\right)$ test with Yates correction. FOLFIRI, 5-fluorouracil, leucovorin and irinotecan.

Based on this analysis the authors concluded that both the toxicity profile and benefits of ILF chemotherapy were similar irrespective of patient age, i.e., below or above 70 years.

Capecitabine monotherapy. Feliu et al (6) assessed the efficacy and tolerability of capecitabine in 51 patients $\geq 70$ years of age as a first-line therapy for metastatic CRC. The treatment was well-tolerated. The initial dose of capecitabine depended on a clearance of creatinine. The toxicity profile (grade $\geq 3$ toxicity) included nausea and vomiting in 1 patient (2\%), diarrhea in 3 patients $(6 \%)$, hand-foot syndrome in 3 patients $(6 \%)$, neutropenia in $1(2 \%)$ patient and thrombocytopenia in $2(4 \%)$ patients. This toxicity was significantly less adverse than in the case of the ILF and LF regimens.

Capecitabine monotherapy resulted in a $24 \%$ objective response rate. The median time to disease progression was 7 months and the median overall survival was 11 months. The results are comparable to those of the LF regimen-based chemotherapy and worse than the results of the ILF regimen.

Cassidy et al (7) evaluated findings of two extensive phase III studies that analyzed the efficacy and safety of oral capecitabine therapy compared with intravenous 5-fluorouracil/ leucovorin. Both age (80 years or older) and creatinine clearance exhibited an impact on the safety profile (increased incidence of grade 3 or 4 gastrointestinal toxicities such as stomatitis and diarrhea, and grade 3 or 4 treatment-related adverse events) of capecitabine therapy $(\mathrm{p}=0.04$ and $\mathrm{p}=0.05$, respectively). A dose reduction in capecitabine was effective in alleviating the toxicities characteristic of infused fluoropyrimidines (stomatitis, diarrhea and hand-foot syndrome). The dose modification of capecitabine was associated wih a minor increase in the risk of disease progression or death and only in patients requiring a dose reduction up to $50 \%$ of the baseline dose [hazard ratio (HR), 1.06; 95\% CI 0.80-1.42; $\mathrm{p}=0.67]$. In patients treated with the 5-fluorouracil/leucovorin regimen, dose reduction was associated with a $30 \%$ increase in the risk of disease progression or death, but was not statistically significant (HR, 1.30; 95\% CI 0.88-1.93; $\mathrm{p}=0.19$ )

Ho et al (8) assessed the efficacy and toxicity of systemic agents in the elderly (aged $\geq 70$ ). The most common first-line chemotherapy regimens were single-agent 5-fluorouracil or capecitabine. Other chemotherapy regimens included oxaliplatin- and irinotecan-based regimens. The overall survival between patients treated with 5 -fluorouracil vs. capecitabine was not statistically significant $(\mathrm{p}=0.65)$. An increased incidence of toxicity was observed in patients treated with 5-fluorouracil vs. capecitabine (43 and 33\%, respectively).

The Danish single centre [Jensen et al (9)] compared the benefits and toxicities of palliative chemotherapy of metastatic CRC based on capecitabine (monotherapy or in combination with oxaliplatin) in 203 non-elderly and 57 elderly patients. No differences were observed between the non-elderly and elderly patients ( $<70$ and $\geq 70$ years) with regard to the objective response rate (33 vs. $37 \%$, respectively) $(\mathrm{p}=0.61)$ and time to disease progression (6.0 vs. 5.5 months, respectively) (HR, 1.09; 95\% CI 0.71-1.68; p=0.84). A difference (trend) was found in overall survival between patients younger and older than 70 years (12.5 vs. 8.4 months, respectively) (HR, 1.48; $95 \%$ CI 1.04-2.38; $\mathrm{p}=0.07)$. More infections $(\mathrm{p}=0.03)$ and neuropathies $(\mathrm{p}=0.02)$ were noted among the younger patients with similar grade 3 or 4 adverse events $(p>0.05)$ in the two groups.

Irinotecan compared to capecitabine. Stec et al (10) retrospectively analyzed the efficacy and tolerability of capecitabine chemotherapy and a FOLFIRI regimen in patients with metastatic CRC over the age of 65 years. No differences in the objective response rate were found between the two analyzed groups. A trend towards a slightly higher overall response rate (28.1\%) [complete response (CR), 9.4\% and partial response (PR), 18.7\%] was found in the group treated with the FOLFIRI regimen compared to patients treated with the capecitabine monotherapy (16.4\%; CR, $4.1 \%$ and PR, 12.3\%); however, this difference did not reach statistical significance $\left(\chi^{2}=2.18\right.$; $\mathrm{p}=0.1398)$. The results are shown in Table III.

Multivariate analysis (Table IV) revealed three independent predictive factors affecting time to disease progression: gender (HR, 0.57; $\mathrm{p}=0.007)$, pretreatment CEA level (HR, 1.81; p=0.012) and location of metastases (HR, 1.66; $\mathrm{p}=0.03$ ). Factors such as type of chemotherapy and Karnofsky performance status had no statistically significant effect. 
Table IV. Multivariate analysis of time to disease progression ${ }^{\mathrm{a}}$.

\begin{tabular}{|c|c|c|}
\hline Covariate & $\mathrm{HR}(95 \% \mathrm{CI})$ & P-value \\
\hline \multicolumn{3}{|l|}{ Gender } \\
\hline Male vs. female & $0.57(0.38-0.86)$ & 0.007 \\
\hline \multicolumn{3}{|l|}{ Location of metastases } \\
\hline Liver vs. other & $1.66(1.05-2.63)$ & 0.030 \\
\hline \multicolumn{3}{|l|}{ Pretreatment CEA level $(\mu \mathrm{g} / \mathrm{l})$} \\
\hline$\leq 5$ vs. $>5$ & $1.81(1.14-2.88)$ & 0.012 \\
\hline \multicolumn{3}{|l|}{ Karnofsky performance status } \\
\hline$\leq 80 \%$ vs. $>80 \%$ & - & $>0.050$ \\
\hline \multicolumn{3}{|l|}{ Chemotherapy } \\
\hline FOLFIRI vs. capecitabine & - & $>0.050$ \\
\hline
\end{tabular}

Multivariate analysis (Table V) revealed prognostic significance of 3 out of 4 factors that were significant predictors in a univariate analysis: gender $(\mathrm{p}=0.00052)$, WHO performance status (HR, 0.51; $\mathrm{p}=0.013)$, and pretreatment CEA level (HR, $3.21 ; \mathrm{p}=0.0001)$. The number of involved organs was not a significant predictor in the multivariate analysis.

Grade 3 and 4 neutropenia was observed more commonly in the group of patients receiving combined chemotherapy vs. monotherapy (grade $3,11.9$ vs. $3.6 \%$; grade $4,7.5$ vs. $0 \%$; grade $3+4,19.4$ vs. $7.5 \%$ ). Other grade 3 and 4 hematological toxicities did not differ between the study groups, i.e., anemia in the FOLFIRI group: grade 3,1.5\%; grade 4, $0 \%$; and capecitabine group: grade $3,1.8 \%$; grade $4,0 \%$. Similarly, no difference was noted for thrombocytopenia in the FOLFIRI group: grade $3+4$, $0 \%$; and the capecitabine group: grade $3,1.8 \%$; grade $4,0 \%$.
Table V. Multivariate analysis of overall survival ${ }^{\text {a }}$

Covariate HR $(95 \% \mathrm{CI}) \quad$ P-value

Primary location

Sigmoid colon vs. colon/rectum $>0.05$

Pretreatment CEA level $(\mu \mathrm{g} / \mathrm{l})$

$\leq 5$ vs. $>5$

3.21 (1.76-5.85)

0.0001

Chemotherapy

FOLFIRI vs. capecitabine

$>0.05$

Age

$\geq 70$ vs. $<70$ years

$>0.05$

Gender

Male vs. female

$0.57(0.36-0.89)$

0.014

WHO performance status

0 vs. $1-2$

$0.51(0.30-0.87)$

0.013

Number of organs involved

1 vs. $\geq 2$

$>0.05$

${ }^{\text {aB }}$ Based on the results by Stec et al (10). CEA, carcinoembryonic antigen; FOLFIRI, 5-fluorouracil, leucovorin and irinotecan; CI, confidence interval; HR, hazard ratio. Bold, statistically significant.

Assessment of grade 3 and 4 non-hematological toxicities showed that hand-foot syndrome was significantly different only in the capecitabine-treated patients (19.6\%). Adverse effects are shown in Table VI.

The analysis was performed retrospectively, and the findings should be viewed in consideration of this limitation. Although the analysis involved more than 120 patients, the sample size may not have been sufficiently large enough to detect differences in efficacy between the two patient cohorts.

Table VI. Toxicities related to capecitabine chemotherapy and a FOLFIRI regimen in patients above 65 years of age (n=123) .

\begin{tabular}{|c|c|c|c|c|c|c|c|c|c|c|c|c|c|}
\hline & \multicolumn{12}{|c|}{ CTC NCI toxicity grade } & \multirow[b]{3}{*}{ P-value } \\
\hline & \multicolumn{4}{|c|}{ I } & \multicolumn{3}{|l|}{ II } & \multicolumn{2}{|l|}{ III } & & \multicolumn{2}{|l|}{ IV } & \\
\hline & $\mathrm{F}$ & & $\mathrm{C}$ & & $\mathrm{F}$ & $\mathrm{C}$ & & $\mathrm{F}$ & $\mathrm{C}$ & & $\mathrm{F}$ & $\mathrm{C}$ & \\
\hline Neutropenia & 10 & $(14.9 \%)$ & 3 & $(5.4 \%)$ & $10(14.9 \%)$ & 2 & $(3.6 \%)$ & $8(11.9 \%)$ & 2 & $(3.6 \%)$ & $5(7.5 \%)$ & - & 0.00003 \\
\hline Anemia & 11 & $(16.4 \%)$ & & $(10.7 \%)$ & $14(20.9 \%)$ & 2 & $(3.6 \%)$ & $1(1.5 \%)$ & 1 & $(1.8 \%)$ & - & - & 0.02000 \\
\hline Thrombocytopenia & 6 & $(8.9 \%)$ & 11 & $(19.6 \%)$ & $1 \quad(1.5 \%)$ & 4 & $(7.1 \%)$ & - & 1 & $(1.8 \%)$ & - & - & 0.07500 \\
\hline Vomiting & 2 & $(3.0 \%)$ & & - & $6 \quad(8.9 \%)$ & 2 & $(3.6 \%)$ & $5(7.5 \%)$ & 3 & $(5.4 \%)$ & - & - & 0.36110 \\
\hline Nausea & 1 & $(1.5 \%)$ & & - & $12(17.9 \%)$ & 2 & $(3.6 \%)$ & $7(10.4 \%)$ & 3 & $(5.4 \%)$ & - & - & 0.06320 \\
\hline Diarrhea & 2 & $(3.0 \%)$ & 1 & $(1.8 \%)$ & $9(13.4 \%)$ & 3 & $(5.4 \%)$ & $4(6.0 \%)$ & 2 & $(3.6 \%)$ & $2(3.0 \%)$ & $1(1.8 \%)$ & 0.25880 \\
\hline Mucositis & 1 & $(1.5 \%)$ & & - & - & 1 & $(1.8 \%)$ & - & 2 & $(3.6 \%)$ & - & - & 0.69690 \\
\hline Asthenia & & - & 1 & $(1.8 \%)$ & $7(10.4 \%)$ & 5 & $(8.9 \%)$ & $9(13.4 \%)$ & 5 & $(8.9 \%)$ & - & $1(1.8 \%)$ & 0.89220 \\
\hline Hand-foot syndrome & & - & 1 & $(1.8 \%)$ & - & & $(14.3 \%)$ & - & 11 & $(19.6 \%)$ & - & - & 0.00070 \\
\hline
\end{tabular}

${ }^{a}$ Based on the results by Stec et al (10). C, capecitabine; F, FOLFIRI: 5-fluorouracil, leucovorin and irinotecan. Bold, statistically significant. 
Table VII. Efficacy of CAPOX in comparison to FUFOX in first-line chemotherapy in patients aged $<70$ and $\geq 70$ years of age .

\begin{tabular}{|c|c|c|c|c|}
\hline & $\begin{array}{c}\text { Age }<70 \text { years } \\
\quad(n=330)\end{array}$ & $\begin{array}{c}\text { Age } \geq 70 \text { years } \\
\quad(n=138)\end{array}$ & $\begin{array}{l}\text { FUFOX } \geq 70 \text { years } \\
\qquad(n=63)\end{array}$ & $\begin{array}{c}\text { CAPOX } \geq 70 \text { years } \\
(n=75)\end{array}$ \\
\hline Median time to disease progression (months) & 7.5 & 7.6 & 7.9 & 7.6 \\
\hline Median overall survival (months) & 18.8 & 14.4 & 14.4 & 14.2 \\
\hline \multicolumn{5}{|l|}{ Response $(\%)$} \\
\hline $\mathrm{CR}+\mathrm{PR}$ & 52 & 49 & 54 & 46 \\
\hline $\mathrm{CR}$ & 5 & 2 & 2 & 3 \\
\hline PR & 47 & 47 & 52 & 43 \\
\hline $\mathrm{SD}$ & 26 & 23 & 19 & 27 \\
\hline
\end{tabular}

aBased on the findings of Arkenau et al (11). CR, complete response; PR, partial response; CR + PR, objective response; SD, stable disease.

Table VIII. Toxicity of FUFOX and CAPOX as first-line chemotherapy in patients aged $<70$ and $\geq 70$ years of age .

\begin{tabular}{|c|c|c|c|c|}
\hline Toxicities of grade 3 and $4(\%)$ & Age $<70$ years & Age $\geq 70$ years & FUFOX $\geq 70$ years & CAPOX $\geq 70$ years \\
\hline Neutropenia & 6 & 9 & 6 & 10 \\
\hline Thrombocytopenia & 1 & 4 & 2 & 5 \\
\hline Anemia & 2 & 4 & 8 & 1 \\
\hline Total & 13 & 20 & - & - \\
\hline Nausea & 8 & 11 & 13 & 9 \\
\hline Diarrhea & 12 & 21 & 22 & 19 \\
\hline Vomiting & 4 & 9 & 11 & 6 \\
\hline Mucositis & 2 & 2 & 3 & 1 \\
\hline Hand-foot syndrome (grade 2/3) & 7 & 8 & 3 & 11 \\
\hline Total & 47 & 45 & - & - \\
\hline
\end{tabular}

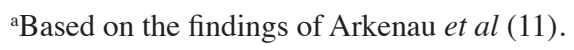

Oxaliplatin. Arkenau et al (11) compared the abovementioned chemotherapeutic regimens in patients with metastatic CRC in a randomized phase III clinical trial. The authors paid particular attention to efficacy and safety of the treatment in patients above 70 years of age who accounted for approximately $30 \%$ of the study population. The analysis consisted of 468 patients, including 138 patients above 70 years of age. The analysis aimed to compare a CAPOX regimen (oxaliplatin $70 \mathrm{mg} / \mathrm{m}$, days 1 and 8; capecitabine 2 x $1000 \mathrm{mg} / \mathrm{m}$; days 1-14 every 3 weeks; after 7 cycles oxaliplatin only on Day 1 to reduce the risk of peripheral neuropathy) and a FUFOX regimen (oxaliplatin $50 \mathrm{mg} / \mathrm{m}, 2-\mathrm{h}$ infusion, leucovorin $500 \mathrm{mg} / \mathrm{m}, 2$-h infusion, 5-fluorouracil $2000 \mathrm{mg} / \mathrm{m}, 22$-h infusion; days 1, 8, 15, 22 every 5 weeks; after 5 cycles oxaliplatin only on Days 1 and 15 to reduce the risk of peripheral neuropathy) in patients older than 70 years of age as well as to compare the two age groups (below and above 70 years of age) which were treated with these chemotherapeutic regimens.

No differences were observed between patients treated with the CAPOX and FUFOX regimens, irrespective of age with regard to the objective response rate (52 vs. 49\%) and time to disease progression (7.5 vs. 7.6 months) (HR, 1.07; 95\% CI 0.86-1.34; $\mathrm{p}=0.54$ ). No difference was found in patients older than 70 years with regard to time to disease progression (7.6 months for CAPOX vs. 7.9 months for FUFOX) and median overall survival (14.2 months for CAPOX vs. 14.4 months for FUFOX). A difference was found in overall survival between patients younger and older than 70 years, with overall suvival being shorter in the latter group (14.4 vs. 18.8 months) (HR, 1.37; 95\% CI 1.07-1.76; $\mathrm{p}=0.03$, Table VII).

The comparison of grade 3 and 4 non-hematological toxicities in patients above than 70 years of age compared to those below 70 years, showed a higher incidence in the former group with regard to diarrhea (21 vs. $12 \%$ ), nausea (11 vs. $8 \%$ ) and vomiting (9 vs. $4 \%$ ), respectively, and a lower incidence of peripheral neuropathy (12 vs. $21 \%$ ), respectively. The comparison of grade 3 and 4 hematological toxicities in the same two groups of patients, showed a higher incidence of neutropenia, anemia and thrombocytopenia in the group of patients above 70 years of age (Table VIII).

Twelves et al (12) compared the efficacy and toxicity of a XELOX regimen between patients older $(n=44)$ and younger $(n=52)$ than 65 years of age. No significant differences were 
Table IX. Dose reduction and treatment discontinuation due to toxicity in patients older and younger than 65 years of age ${ }^{a}$.

\begin{tabular}{lcc}
\hline & \multicolumn{2}{c}{ Patient age } \\
\cline { 2 - 3 } & $\begin{array}{c}<65 \text { years } \\
(\mathrm{n}=52)\end{array}$ & $\begin{array}{c}\geq 65 \text { years } \\
(\mathrm{n}=44)\end{array}$ \\
\hline Capecitabine & $18(35 \%)$ & $18(41 \%)$ \\
$\quad \begin{array}{l}\text { Dose reduction } \\
\text { Median time to dose reduction }\end{array}$ & 76 days & 90 days \\
Oxaliplatin & $17(33 \%)$ & $17(39 \%)$ \\
$\quad \begin{array}{l}\text { Dose reduction } \\
\text { Median time to dose reduction }\end{array}$ & 106 days & 90 days \\
Treatment discontinuation due to & $10(19 \%)$ & $6(14 \%)$ \\
toxicity & 0 & $3(7 \%)^{\mathrm{b}}$ \\
Death & 0 & \\
\hline
\end{tabular}

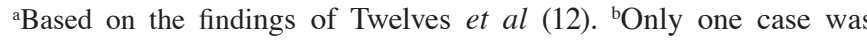
treatment-related.

found between the two groups with regard to time to disease progression $(\mathrm{p}=0.85)$ and overall survival $(\mathrm{p}=0.65)$. A higher objective response rate of $58 \%$ (95\% CI $43-71$ ) vs. $52 \%$ (95\% CI 37-69) and a stable disease rate of $35 \%$ (95\% CI 22-49) compared to $27 \%$ (95\% CI 15-43) were found for younger patients compared to ones older than 65 years of age. No significant differences were found for grade 3 and 4 toxicities between patients younger and older than 65 years of age, apart from hand-foot syndrome which was observed only in patients younger than 65 years of age. The percentage of patients in whom drug doses were reduced or the treatment was discontinued due to toxicity was also comparable in the two patient groups (Table IX).

Feliu et al (13) assessed the efficacy and tolerance of the chemotherapeutic regimen XELOX in 50 patients who were 70 years or older as a first-line therapy for metastatic CRC. The median time to disease progression was 5.8 months (95\% CI 3.9-7.8) and the median overall survival was 13.2 months (95\% CI 7.6-16.9). In this group of patients, the objective response rate was $36 \%$ (95\% CI 28-49), the stable disease rate was 36\% (18 patients) and disease progression was noted in $14(28 \%)$ patients. The treatment was well-tolerated. Grade $\geq 3$ toxicities were found in $14(28 \%)$ patients: in $11(22 \%)$ diarrhea, in $8(16 \%)$ weakness, in $7(14 \%)$ nausea/vomiting, in $3(6 \%)$ neutropenia, in $3(6 \%)$ thrombocytopenia and in $2(4 \%)$ hand-foot syndrome.

Findings of an analysis of 1408 patients (213 patients above 70 years of age) regarding the efficacy/safety of a FOLFOX regimen were reported by Tabah-Fisch et al (14). Particular attention was paid to the efficacy and safety of the treatment in patients above 70 years of age. The analysis involved a comparison of a FOLFOX regimen (adjuvant, first-line and second-line chemotherapy) in younger and older patients. Neutropenia, thrombocytopenia, stomatitis, diarrhea slightly increased with age ( $<70$ vs. $\geq 70$ years of age), but overall severe toxicities were found to be similar. It was noted that the efficacy (response rates, progression-free survival and overall survival in first- and second-line chemotherapy) was similar in the two groups.

Goldberg et al (15) retrospectively analyzed the efficacy and tolerability of a FOLFOX 4 regimen vs. the control from four trials in adjuvant, first-line and second-line settings. This analysis included 3742 patients with CRC (614 aged $\geq 70$ years). The analysis documented similar benefits in the two groups ( $<70$ vs. $\geq 70$ years of age) in terms of response rates, progression or relapse-free survival (HR, 0.70 for FOLFOX 4 vs. control for age $<70$ years and HR, 0.65 for FOLFOX 4 vs. control for age $\geq 70$ years; $p=0.42$ ) and overall survival (HR, 0.77 for FOLFOX 4 vs. the control for age $<70$ years and HR, 0.82 for FOLFOX 4 vs. the control for age $\geq 70$ years; $p=0.79$ ).

A significantly higher incidence of neutropenia grade $\geq 3$ (43 vs. $49 \%, \mathrm{p}=0.04$ ) and thrombocytopenia grade $\geq 3$ ( 2 vs. $5 \%, p=0.04$ ) was noted in the older compared to the younger patients.

In the pooled analysis, the criterion of older age did not increase the rates of gastrointestinal toxicities (nausea, vomiting and diarrhea; 20 vs. $20 \%, p=0.38$ ), neurotoxicity (12 vs. $14 \%, \mathrm{p}=0.37$ ), infection ( 5 vs. $4 \%, \mathrm{p}=0.57$ ), fatigue ( 4 vs. $7 \%, p=0.08$ ) and incidence of grade $\geq 3$ toxicity ( 63 vs. $67 \%, \mathrm{p}=0.15)(15,16)$.

\section{Targeted therapies}

Bevacizumab. A significant phase III clinical study (BICC-C study: bolus, infusional, or capecitabine with camptostarcelecoxib) was performed by Jackson et al (17). This study utilized chemotherapy alone (1st study period) and bevacizumab in combination with chemotherapy (2nd study period). Assessment of the efficacy and toxicity of the regimens: FOLFIRI, mIFL (irinotecan plus 'bolus' 5-fluorouracil/ leucovorin), CAPIRI (irinotecan plus capecitabine), FOLFIRI plus bevacizumab and mIFL plus bevacizumab in patients younger and older than 70 years of age was an important aspect of this study. Apart from chemotherapy and chemoimmunotherapy, the patients received celecoxib or a placebo, depending on randomization. A total of 430 patients (84 patients older than 70 years of age) were enrolled in the 1st study period and 117 (29 patients older than 70 years of age) were enrolled in the 2 nd study period. When treatment efficacy in the 1st study period (chemotherapy alone) was assessed, the median time to disease progression was comparable in patients younger and older than 70 years of age (6.6 vs. 7.5 months; HR, 0.98 ; 95\% CI 0.74-1.29). The median overall survival was longer in patients older than 70 years (19 vs. 21.2 months, respectively; HR, 1.15; 95\% CI $0.87-1.51$ ). In the 2nd study period (chemotherapy combined with immunotherapy), the median time to disease progression and median overall survival were longer in patients younger than 70 years of age compared to those above 70 years of age (10.6 vs. 7.6 months; HR, 1.78 ; 95\% CI 0.93-3.41 and 25.1 vs. 19.4 months; HR, 1.41; 95\% CI 0.83-2.41) (Table X).

With regard to a comparison of hematological and nonhematological grade 3 and 4 toxicities in the patients older compared to younger than 70 years of age, dehydration and weakness were more common while the incidence of other toxicities was comparable in the two patient groups (Table XI). 
Table X. Efficacy of chemotherapy and chemoimmunotherapy in patients younger and older than 70 years of age .

$\begin{array}{ccc}\begin{array}{c}\text { Patient age }<70 \text { years } \\ (n=346)\end{array} & \text { Patient age } \geq 70 \text { years } & \text { HR }(95 \% \mathrm{CI}) \\ (\mathrm{n}=84) & \end{array}$

\begin{tabular}{|c|c|c|c|}
\hline Median time to disease progression (months) & $6.6(6.0-7.1)$ & $7.5(5.9-8.6)$ & $0.98(0.74-1.29)$ \\
\hline Median overall survival (months) & $19.0(17.2-23.2)$ & $21.2(14.2-23.7)$ & $1.15(0.87-1.51)$ \\
\hline \multirow[t]{2}{*}{ Complete response rate $(\%)$} & 47 & 50 & \\
\hline & $\begin{array}{c}\text { Patient age }<70 \text { years } \\
\qquad(\mathrm{n}=88)\end{array}$ & $\begin{array}{l}\text { Patient age } \geq 70 \text { years } \\
\qquad(\mathrm{n}=29)\end{array}$ & $\operatorname{HR}(95 \% \mathrm{CI})$ \\
\hline \multicolumn{4}{|l|}{ 2nd study period (chemoimmunotherapy) } \\
\hline Median time to disease progression (months) & $10.6(8.5-13.8)$ & $7.6(4.3-17.4)$ & $1.78(0.93-3.41)$ \\
\hline Median overall survival (months) & $25.1(19.8-30.5)$ & $19.4(11.6-26.6)$ & $1.41(0.83-2.41)$ \\
\hline \multicolumn{4}{|l|}{ Complete response rate $(\%)$} \\
\hline FOLFIRI/bevacizumab & 58 & 57 & \\
\hline mIFL/bevacizumab & 58 & 40 & \\
\hline
\end{tabular}

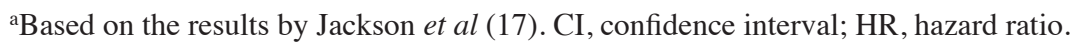

Table XI. Toxicity of chemotherapy and chemoimmunotherapy in patients younger and older than 70 years of age ${ }^{a}$.

\begin{tabular}{lrcr}
\hline & \multicolumn{2}{c}{ Patient age } & \\
\cline { 2 - 3 } Toxicity of grade $\geq 3(\%)$ & $<70$ years & $\geq 70$ years & P-value \\
\hline Leucopenia & 21 & 27 & 0.290 \\
Neutropenia & 37 & 46 & 0.130 \\
Neutropenic fever & 8 & 6 & 0.520 \\
Nausea & 11 & 12 & 0.820 \\
Diarrhea & 26 & 30 & 0.470 \\
Deep vein thrombosis & 7 & 8 & 0.720 \\
Fatigue & 11 & 10 & 0.810 \\
Dehydration & 9 & 18 & 0.020 \\
Weakness & 3 & 11 & 0.001 \\
Dose reduction & 17 & 13 & 0.390 \\
\hline
\end{tabular}

${ }^{\mathrm{a} B a s e d}$ on the results by Jackson et al (17).

Cetuximab. Gràvalos et al (18) analyzed the efficacy and tolerability of cetuximab in combination with capecitabine. A total of 66 patients aged $>70$ years were included in the study. The analysis cohort was divided into two groups according to the respective regimens. Group A received cetuximab $400 \mathrm{mg} /$ $\mathrm{m}^{2}$ for the initial dose and subsequently $250 \mathrm{mg} / \mathrm{m}^{2}$ weekly; capecitabine $1250 \mathrm{mg} / \mathrm{m}^{2}$ twice daily was administered per os for 14 days followed by a 7-day rest every 21 days. Group B received cetuximab $400 \mathrm{mg} / \mathrm{m}^{2}$ for the initial dose and subsequently $250 \mathrm{mg} / \mathrm{m}^{2}$ weekly; capecitabine $1000 \mathrm{mg} / \mathrm{m}^{2}$ twice daily was administered for 14 days followed by a 7-day rest every 21 days. In the case of toxicity, a reduction in drug doses was permitted.

The rates of overall objective responses (complete plus partial) were similar in the two groups: $32 \%$ in group A and $35 \%$ in group B. The rate of disease stabilization in group A (41\%) was lower than that in group B $(58 \%)$. The preliminary results suggest higher activity of the combination therapy than cetuximab alone (overall response, 14.6\%) (19).

Toxicity grade 3-4 (nail toxicity, diarrhea and hand-foot syndrome) occurred with a higher incidence in the patients treated with a higher dose of capecitabine (group A: 32, 14 and $18 \%$, respectively) than in those treated with a lower dose of capecitabine (group B: 7, 9 and 5\%, respectively). Acne-like rash grade 3-4 was similar in the two groups: $23 \%$ in group A and $25 \%$ in group B. Of the patients in group A $27 \%$ discontinued treatment due to adverse events, while only $9 \%$ patients in group B discontinued treatment.

Panitumumab. Results of a phase III study (PACCE; Panitumumab Advanced Colorectal Cancer Evaluation) which evaluated the efficacy and safety of bevacizumab and chemotherapy (oxaliplatin- and irinotecan-based) with or without panitumumab were reported by Hecht et al (20). This study included 1053 patients (823 in the oxaliplatin and 230 in the irinotecan group), including 416 patients above 65 years of age. Following an interim analysis of 812 patients in the oxaliplatin group, panitumumab was discontinued. In the final analysis, the median progression-free survival was 10.0 months in patients treated with panitumumab and 11.4 months in patients treated in the control arms (without panitumumab) (HR, 1.27; 95\% CI 1.06-1.52), and median survival was respectively 19.4 and 24.5 months (HR, 1.43; 95\% CI 1.11-1.83). Grade 3 and 4 skin toxicity, diarrhea, infections and pulmonary embolism 
were more frequent in the panitumumab group than in the control group (36 vs. $1 \% ; 24$ vs. $13 \% ; 19$ vs. $10 \% ; 6$ vs. $4 \%$, respectively).

\section{Conclusions}

Considerable progress has been made in the treatment of colorectal cancer due to the implementation of new oncological drugs that have resulted in a significant prolongation of survival of patients with stage IV disease (from 4.6 months for best supportive care to 20.6 months for new chemotherapeutic regimens) $(21,22)$. Physicians are able to use new therapies; however, this use is limited by significant adverse effects that reduce the safety of the therapy and impair the quality of life of the patient. This obstacle is particularly evident in patients aged 65 years or older for whom the chemotherapy-related risk is high, partially related to decreased organ performance and significant co-morbidities (e.g. ischemic heart disease, diabetes mellitus, hypertension and lung disease).

Age is a significant factor affecting clearance of creatinine. Lower creatinine clearance was found to require dose modification in patients treated with capecitabine. In metastatic colorectal cancer patients aged 80 years or older treated with capecitabine or a 5-fluorouracil/leucovorin regimen, the toxicity risk was enhanced with an increased incidence of grade 3 or 4 treatment-related adverse effects. Although a dose reduction in capecitabine was effective in alleviating toxicities characteristic of infused fluoropyrimidines (stomatitis, diarrhea and hand-foot syndrome), the dose modification of capecitabine was associated with a minor increase in the risk of disease progression or death. In patients treated with a 5-fluorouracil/leucovorin regimen, dose reduction did not significantly affect the increase in the risk of disease progression or death (7).

A retrospective analysis (969 patients) also showed that patients aged 80 years or older with stage II or III colon cancer benefit from adjuvant chemotherapy. The overall 5 -year survival rates for stage II were $63 \%$ for patients with adjuvant chemotherapy compared to $36 \%$ without treatment (HR, 0.536; $\mathrm{p}<0.0097$ ) and for stage III, 54 compared to $20 \%$, respectively (HR, 0.424; p=0.0001) (23).

Analysis of the efficacy of the presented chemotherapeutic and chemoimmunotherapeutic regimens in the treatment of metastatic colorectal cancer in patients older than 65 and 70 compared to 'younger' patients, showed generally comparable efficacy with regard to both time to disease progression and overall survival. No significant differences in the incidence of grade 3 and 4 toxicities were found between these patient groups despite expected poorer treatment tolerance in 'older' patients. Notably, a retrospective comparison of capecitabine monotherapy and the FOLFIRI regimen did not show any differences in treatment efficacy between the two therapeutic options. This finding should be confirmed in prospective, randomized clinical trials. The systemic treatment (treatment regimen) should be based on the results and toxicity of the particular clinical trials.

Based on these investigations, elderly patients are favorable candidates for first-line chemotherapy such as an oxaliplatinor irinotecan-based regimen, but the safety of adding targeted agents to chemotherapy warrants further research (24), apart from monotherapy of panitumumab or cetuximab $(25,26)$. More patients are eligible for second-line and even third-line chemotherapy.

In conclusion, the age criterion should not be considered when assessing the eligibility of patients with metastatic colorectal cancer for treatment. The treatment should be individualized based on the potential risks and benefits anticipated for each patient. Such assessment of eligibility should be based primarily on the evaluation of performance status and the presence of co-morbidities.

\section{References}

1. Ries LAG, Eisner MP, Kosary CL, et al: SEER Cancer Statistics Review 1973-1998. National Cancer Institute, Bethesda, MD, 2001.

2. National Institutes of Health: Surveillance epidemiology and end results: Available at: http://seer.cancer.gov/, 2008.

3. Adjei AA: A review of the pharmacology and clinical activity of new chemotherapy agents for the treatment of colorectal cancer. J Clin Pharmacol: 48: 265-277, 1999.

4. Lichtman SM and Villani G: Chemotherapy in the elderly: pharmacologic considerations. Cancer Control 7: 548-556, 2000.

5. Folprecht G, Seymour MT, Saltz L, et al: Irinotecan/fluorouracil combination in first-line therapy of older and younger patients with metastatic colorectal cancer: combined analysis of 2,691 patients in randomized controlled trials. J Clin Oncol 26: 1443-1451, 2008.

6. Feliu J, Escudero P, Llosa F, et al: Capecitabine as first-line treatment for patients older than 70 years with metastatic colorectal cancer: an Oncopaz Cooperative Group study. J Clin Oncol 23: 3104-3111, 2005.

7. Cassidy J, Twelves C, Van Cutsem E, et al: First-line oral capecitabine therapy in metastatic colorectal cancer: a favorable safety profile compared with intravenous 5-fluorouracil/ leucovorin. Ann Oncol 13: 566-575, 2002.

8. Ho C, Ng K, O'Reilly S and Gill S: Outcomes in elderly patients with advanced colorectal cancer treated with capecitabine: a population-based analysis. Clin Colorectal Cancer 5: 279-282, 2005.

9. Jensen SA, Lonborg JT and Sorensen JB: Benefits and risk of palliative capecitabine-based therapy to elderly patients with advanced colorectal cancer: Danish single center experiences. Acta Oncol 45: 67-76, 2006.

10. Stec R, Bodnar L and Szczylik C: Feasibility and efficacy of capecitabine and FOLFIRI in patients aged 65 years and older with advanced colorectal cancer: a retrospective analysis. J Cancer Res Clin Oncol 136: 283-292, 2010.

11. Arkenau HT, Graeven U, Kubicka S, et al: Oxaliplatin in combination with 5-fluorouracil/leucovorin or capecitabine in elderly patients with metastatic colorectal cancer. Clin Colorectal Cancer 7: 60-64, 2008.

12. Twelves CJ, Butts CA, Cassidy J, et al: Capecitabine/oxaliplatin, oxaliplatin a safe and active first-line regiment for older patients with metastatic colorectal cancer: post hoc analysis of a large phase II Study. Clin Colorectal Cancer 5: 101-107, 2005.

13. Feliu J, Salud A, Escudero P, et al: XELOX (capecitabine plus oxaliplatin) as first-line treatment for patients over 70 years of age with advanced colorectal cancer. Br J Cancer 94: 969-975, 2006.

14. Tabah-Fisch I, Maindrault-Goebel F, Benavides M, et al: Oxaliplatin/5 FU/LV is feasible, safe and active in elderly colorectal cancer (CRC) patients. ASCO abs. 556, 2002.

15. Goldberg RM, Tabah-Fisch I, Bleiberg H, et al: Pooled analysis of safety and efficacy of oxaliplatin plus fluorouracil/lecovorin administered bimonthly in elderly patients with colorectal cancer. J Clin Oncol 24: 4085-4091, 2006.

16. Bruce C, Köhne C-H and Audisio RA: Treatment of advanced colorectal cancer in the elderly. Eur J Surg Oncol: 84-87, 2007.

17. Jackson NA, Barrueco J, Soufi-Mahjoubi R, et al: Comparing safety and efficacy of first-line irinitecan/fluorropyrimidine combinations in eldery versus nonelderly patients with metastatic colorectal cancer. Cancer 115: 2617-2629, 2009. 
18. Gràvalos $\mathrm{C}$, River $\mathrm{F}$, Massuti $\mathrm{B}$, et al: Cetuximab and capecitabine as first-line treatment for elderly patient (pts) with metastatic colorectal cancer $(\mathrm{mCRC})$ : preliminary results of TTD trial. (suppl) J Clin Oncol 26, 2008.

19. Sastre J, Aranda E, Gràvalos C, et al: First-line single-agent cetuximab in elderly patients with metastatic colorectal cancer. A phase II clinical and molecular study of the Spanish Group for Digestive Tumor Therapy (TTD). Crit Rev Oncol Hematol: Dec. 28, 2009 (Epub ahead of print).

20. Hecht JR, Mitchell E, Chidiac T, et al: A randomized phase IIIB trial of chemotherapy, bevacizumab, and panitumumab compared with chemotherapy and bevacizumab alone for metastatic colorectal cancer. J Clin Oncol 27: 672-680, 2009.

21. Wolpin BM and Mayer RJ: Systemic treatment of colorectal cancer. Gastroenterology 134: 1296-1310, 2008.

22. Saltz L, Clarke S, Diaz-Rubio E, et al: Bevacizumab (Bev) in combination with XELOX or FOLFOX4: updated efficacy results from XELOX-1/NO16966, a randomized phase III trial in first line metastatic colorectal cancer. J Clin Oncol 25: 18S, 2007.
23. Sama A, Gonsalves W, Aldoss I, et al: Adjuvant chemotherapy for colon cancer patients age 80 and older: Veteran's Affairs Central Cancer (VACCR) database analysis. Gastrointestinal Cancers Symposium ASCO abs. 450, 2010.

24. Köhne C-H, Folprecht G, Goldberg RM, et al: Chemotherapy in elderly patients with colorectal cancer. Oncologist 13: 390-402, 2008.

25. Amado RG, Wolf M, Peeters M, et al: Wild-type KRAS is required for panitumumab efficacy in patients with metastatic colorectal cancer. J Clin Oncol 26: 1626-1634, 2008.

26. Karapetis CS, Khambata-Ford S, Jonker DJ, et al: K-ras mutations and benefit from cetuximab in advanced colorectal cancer. N Engl Med 359: 1757-1765, 2008. 